\title{
Interview with Paul-Peter Tak: Stimulating the vagus nerve to treat rheumatoid arthritis
}

\author{
Professor Paul-Peter Tak ${ }^{*}, 1,2,3,4$ \\ ${ }^{1}$ Academic Medical Center, University of Amsterdam, Amsterdam, The Netherlands \\ ${ }^{2}$ Cambridge University, Cambridge, UK \\ ${ }^{3}$ Ghent University, Ghent, Belgium \\ ${ }^{4}$ GSK Medicines Research Centre, Gunnels Wood Road, Stevenage, Hertfordshire, SG1 2NY, UK \\ * Author for correspondence: p.p.tak@amc.uva.nl
}

First draft submitted: 6 July 2017; Accepted for publication: 15 November 2017; Published online: 14 December 2017

\section{How did you become interested in the autonomic nervous system \& immune-mediated inflammatory diseases?}

To explain the story briefly, I became interested in the cholinergic and anti-inflammatory pathway due to serendipity. We had previously carried out a screen using fibroblast-like synoviocytes derived from arthroscopic synovial tissue samples from the joints of rheumatoid arthritis (RA) patients. We isolated these key effector cells in the joint (the fibroblast-like synoviocytes) and used high-throughput assays to screen adenoviral vectors expressing shRNA coding for 807 transcripts to look for novel therapeutic targets in chronic immune-mediated inflammatory diseases (IMIDs). As a read-out we looked at the production of IL-8, which is a key cytokine and chemokine in the pathogenesis of RA, and also matrix metalloproteinases (MMPs), which are important degrading enzymes.

We used this approach as a screen and had several hits, one of which was, to my surprise, the alpha-7 nicotinic acetylcholine receptor. We then carried out additional work to test the effects of alpha-7 nicotinic acetylcholine receptor agonists in vitro and observed that if you stimulate the alpha-7 receptor in the fibroblast-like synoviocytes it leads to reduced production of IL-8 and pro-inflammatory cytokines such as IL-6 as well as a variety of MMPs.

\section{Could you briefly describe your own work that led you to hypothesise that electrical stimulation of the vagus nerve could have an anti-inflammatory effect in RA patients?}

We wanted to explore the role of the alpha-7 receptor in vivo using an animal model of rheumatoid arthritis. This was basically the first work on the role of the cholinergic enzyme family pathway and the vagus nerve in a chronic model of inflammation.

Using the collagen-induced arthritis model, which is an established model of (RA), in alpha-7 knockout mice, we found that these mice have increased arthritis, increased synovitis shown by histology, and increased destruction of bone and cartilage when compared with matched controls. Following this we performed the opposite experiment in that we stimulated this pathway by injecting the collagen-induced arthritis mice (with a normal background) with nicotine which stimulates the alpha-7 receptor. We did this ip and orally, and found reduced arthritis, and reduced bone and cartilage degradation. We then tested several alpha-7 specific agonists and found exactly the same. We also did an experiment in these mice in which we carried out a hemilateral vagotomy and observed that there was increased arthritis activity.

Taken together, so far we had found that in vitro, using human cells, if you stimulate the alpha-7 receptor it leads to reduced production of mediators of inflammation and degradation. In the different animal models that we tested we found that if you disrupt the cholinergic pathway by unilateral vagotomy or by knocking out the alpha-7 receptor you can increase arthritis and, in contrast, if you stimulate this pathway by using nicotine or alpha-7 specific agonists you find reduced arthritis.

Based on the results of these experiments we proposed in a 2009 review article in Nature Reviews Rheumatology that it may be possible to stimulate this pathway using either small molecules to activate the alpha-7 receptor or by a bioelectronic approach using electrical stimulation of the vagus nerve [1].

This work is closely linked to the pioneering studies in acute inflammation conducted by Kevin Tracey, who has termed the mechanism by which the vagus nerve may sense inflammation and subsequenbtly inhibit it the 'the 
inflammatory reflex'. We were however interested in in vitro and animal models of chronic inflammation to ask the question: could this be relevant for the treatment of chronic immune-mediated inflammatory disorders?

We began a conversation with a small company in the USA called SetPoint and we worked with them to stimulate the vagus nerve with an electrical signal in rats with collagen-induced arthritis, another model of RA. We found that if the vagus nerve was stimulated for only 60s per day that this translates into clinical improvement in the rats and actually also into protection against progressive joint destruction.

\section{Your team trialled VNS in RA patients. Could you briefly describe the results in terms of outcome for patients?}

We worked with SetPoint, using a commercially available device from Cyberonics, which has been approved for the treatment of therapy-resistant epilepsy and is also used in therapy-resistant depression. More than 100,000 patients at the time had been treated with this Cyberonics device, so there were a lot of safety data available. Now, we can work in in vitro models and animal models forever, but I'm a physician-scientist so I want to know whether this might actually be relevant in humans.

In order to evaluate this we moved to a mechanistic, experimental medicine clinical trial in RA patients to learn whether this approach could be relevant as a new treatment option. If this proved to be the case then we could take it back into animal models for optimization.

SetPoint was willing to sponsor and support this clinical trial with the device from Cyberonics. I designed the clinical trial based on our extensive experience with the evaluation of new treatments, using relatively small, biomarker-rich clinical studies for early go/no go decisions, and served as the principal investigator for this international study. It consisted of two stages. First, we tested vagus nerve stimulation in RA patients who had previously failed conventional disease-modifying antirheumatic drugs (DMARDs). We found in seven patients that if you stimulate for 60s per day, and in those that did not respond initially we increased the time to 4 mins per day divided over the day, there was very clear improvement in a subset of the patients as measured with the disease activity score evaluated in 28 joints (DAS 28), a validated clinical end point.

And then we thought, we can go even further and test this concept in patients with therapy-resistant disease. This is the second cohort of ten patients who had not only failed conventional DMARDs but also two biopharmaceuticals with a different mechanism of action. Again we found that, although there is no treatment that works in all patients which is probably explained by the fact that RA is more like a syndrome rather than a well-defined single disease, in this patient cohort very remarkable responses were observed and even, in some cases, very durable responses. Patients achieved remission, in other words absence of disease activity, and some of these patients were able to stop taking background medication.

If you want to get a feel for these patients you can find the presentation of one of them online. In the interview on Sky news, the patient describes how much she has improved; it is also interesting that during the interview she is stimulating the vagus nerve and you hear a slight change in her voice.

This is not the only patient who has responded this way. Recently, during the PhD ceremony of one of my students, I met another patient, the first person who was ever treated with vagus nerve stimulation for RA. She also told me that she is in stable remission after several years now. Although that is obviously not achieved in all patients, but in a minority, it's very remarkable. I'm a rheumatologist with a lot of clinical experience and I can tell you that it's very unusual for an RA patient who has failed multiple DMARDs and biopharmaceuticals to be able to achieve stable disease remission in the absence of any background medication. There is much more than anedectotal reports, and for the data I refer to our paper.

We also tested a small group of patients who had the same vagus nerve device implanted for the treatment of epilepsy. We found in these patients that if you take blood samples at three time points: before implantation of the device, after implantation while they are still under anesthesia (before vagus nerve stimulation), and the third after stimulation, there is a significant reduction in LPS-induced TNF production and also in the serum levels of IL-6 and IL-1. In other words, we could show, in patients who were stimulated under anesthesia (so there can't be a placebo effect) that even a single stimulation of the vagus nerve results in a decrease in the production of pro-inflammatory cytokines known to be intimately involved in the pathogenesis of conditions like RA.

For those who want to hear more, the data from the human trial was published in PNAS in 2016 [2] and the work in animal models has been published over the last eight years or so. We published the hypothesis that this might be a new treatment for RA in Nature Reviews Rheumatology [1]. More recently we summarized the field and our contribution to it in the Journal of Internal Medicine [3]. 


\section{Could you speculate on the mechanisms behind the effect of VNS on inflammation in RA \& any other conditions VNS stimulation could be applied to?}

I'm very interested in looking at the different stages of diseases like RA, because there may be important lessons about the pathogenesis. The same way of thinking about different phases is probably relevant for other conditions, like Type I diabetes, which is basically pancreatic autoimmunity, or multiple sclerosis. We and others did a lot of work on the earliest stages of RA, and found that individuals who are at risk of developing RA can be identified before they develop arthritis based on their autoantibody profile. These people may have rheumatoid factor, which is an autoantibody, and/or anti-citrullinated peptide antibodies, which you can measure with for example the anti-CCP test. If these autoantibodies are present, subjects have a chance of developing RA of approximately $30-40 \%$ in 2 years' time in our cohorts.

We examined two such cohorts and measured different parameters before the individuals developed arthritis including vagal nerve tone, which can be done by looking at resting heart rate and heart rate variability. We found in this cohort, and this was confirmed in an independent cohort, that decreased vagus nerve tone precedes the development of RA in at-risk populations based on the autoantibody profile. As not everybody will develop RA, this is an independent risk factor for its development. This suggests that inflammation in RA, which is a complex autoimmune disease that is influenced by multiple mechanisms, can be controlled by vagus nerve activity. These data support the notion that restoration of the dysbalance in the autnomous nervous system, as observed in RA, by vagus nerve stimulation using a bioelectronics approach will have a beneficial effect on disease activity.

The mechanisms behind the effect of vagus nerve stimulation on inflammation are complex. Based on Kevin Tracey's groundbreaking experiments, the current evidence suggests that vagus nerve stimution leads to increased splenic nerve activity, followed by increased release of acetylcholine by splenic $T$ cells that interact with monocytes and macrophages. As a result there is a decrease in the production of pro-inflammatory cytokines by these cells.

The data in pre-RA cohorts really points towards a causal role for abnormalities in the autonomic nervous system that play a role in the development of RA. This is not restricted to RA but is most likely relevant to a whole variety of diseases. In terms of the therapeutic effect that we have seen in the trials, I know of two different small studies in Crohn's disease that support our findings. For instance, in a study in Amsterdam in six patients who were inadequate responders to anti-TNF therapies there was clincial and endoscopic improvement aftre vagus nerve stimulation. There is also a separate small study from France, showing exactly the same. This gives me confidence about the scientific story we have based on in vitro studies, preclinical models and investigations in humans, which are now being confirmed by others.

\section{How do you think the field will develop over the next 5-10 years?}

The work I discussed here is based on my research as a professor of rheumatology in Amsterdam. At GSK we are also interested in bioelectronic medicine. GSK formed a company together with Verily Life Sciences (Google's life sciences company) called Galvani Bioelectronics that will focus on the development bioelectronic medicines. They will make significant investments to develop miniaturised, implantable devices for a variety of diseases. It's a clear illustration of the fact that very big companies are convinced that bioelectronic medicine is becoming really important and I think for a company like GSK the mission is to improve the life of patients by whatever modality and this is not necessarily restricted to the use of small molecules or by the use of biopharmaceuticals or even gene therapy. As a company we will go where the science will take us, and I predict that we may become increasingly a bioelectronics company over time. There are many other initiatives in this field as well, SetPoint are pursuing their efforts in the field of bioelectronic treatment of patients with chronic inflammatory disorders. In the next 10-15 years I do believe that there will be subsets of patients that will be treated with electrodes rather than with chemicals.

\section{Financial \& competing interests disclosure}

Paul-Peter Tak is an employee of GlaxoSmithKline, which holds an equity interest in SetPoint Medical Corporation. He is also a Board Member of Galvani Bioelectronics. The author has no other relevant affiliations or financial involvement with any organization or entity with a financial interest in or financial conflict with the subject mateter or materials discussed in the manuscript apart from those disclosed.

No writing assistance was utilized in the production of this manuscript. 


\section{References}

1. van Maanen MA, Vervoordeldonk MJ, Tak PP. The cholinergic anti-inflammatory pathway: towards innovative treatment of rheumatoid arthritis. Nat. Rev. Rheumatol. 5(4), 229-232 (2009).

2. Koopman FA, Chavan SS, Miljko $S$ et al. Vagus nerve stimulation inhibits cytokine production and attenuates disease severity in rheumatoid arthritis. Proc. Natl Acad. Sci. USA 113(29), 8284-8289 (2016).

3. Koopman FA, van Maanen MA, Vervoordeldonk MJ, Tak PP. Balancing the autonomic nervous system to reduce inflammation in rheumatoid arthritis. J. Intern. Med. 282(1), 64-75 (2017). 